\title{
Soliton bunching in annular Josephson junctions
}

Vernik, I.V; Lazarides, Nickos; Sørensen, Mads Peter; Ustinov, A.V.; Pedersen, Niels Falsig; Oboznov, V.A.

Published in:

Journal of Applied Physics

Link to article, DOI:

10.1063/1.362394

Publication date:

1996

Document Version

Publisher's PDF, also known as Version of record

Link back to DTU Orbit

Citation (APA):

Vernik, I. V., Lazarides, N., Sørensen, M. P., Ustinov, A. V., Pedersen, N. F., \& Oboznov, V. A. (1996). Soliton bunching in annular Josephson junctions. Journal of Applied Physics, 79(10), 7854-7859.

https://doi.org/10.1063/1.362394

\section{General rights}

Copyright and moral rights for the publications made accessible in the public portal are retained by the authors and/or other copyright owners and it is a condition of accessing publications that users recognise and abide by the legal requirements associated with these rights.

- Users may download and print one copy of any publication from the public portal for the purpose of private study or research.

- You may not further distribute the material or use it for any profit-making activity or commercial gain

- You may freely distribute the URL identifying the publication in the public portal

If you believe that this document breaches copyright please contact us providing details, and we will remove access to the work immediately and investigate your claim. 


\title{
Soliton bunching in annular Josephson junctions
}

\author{
I. V. Vernik, ${ }^{a), b)}$ N. Lazarides, M. P. Sørensen, A. V. Ustinov, ${ }^{c)}$ N. F. Pedersen, \\ and V. A. Oboznov) \\ Physics Department and Institute of Mathematical Modelling, The Technical University of Denmark, \\ DK-2800 Lyngby, Denmark
}

(Received 1 December 1995; accepted for publication 5 February 1996)

\begin{abstract}
By studying soliton (fluxon) motion in long annular Josephson junctions it is possible to avoid the influence of the boundaries and soliton-soliton collisions present in linear junctions. A new experimental design consisting of a niobium coil placed on top of an annular junction has been used to insert individual fluxons or antifluxons into the junction in a controllable way. The dynamical behavior of different numbers of trapped fluxons was investigated. In addition, we were able to change the junction parameters by changing temperature. In some of the zero-field steps, the experiments reveal a small jump to higher values of the voltage at the top of the steps. This phenomenon can be caused by a crossover from a nonbunched state to a bunched state of the fluxon motion. By performing direct numerical simulations using the perturbed sine-Gordon equation with parameter values determined from the experiments we have been able to confirm the above explanation. (C) 1996 American Institute of Physics. [S0021-8979(96)03810-2]
\end{abstract}

\section{INTRODUCTION}

After the construction of the first annular long Josephson junction $(\mathrm{LJJ})^{1}$ it has been become possible to study motion of solitons (fluxons) which is not disturbed by boundaries. In the conventional LJJ with linear geometry the analysis of experimental results is complicated due to reflections from boundaries and collisions among the fluxons. The annular LJJ offers the possibility of studying fluxon motion experimentally in a clean case. Numerical simulations, ${ }^{2}$ which include surface losses, show that there exists a critical value of the bias current above which two solitons may bunch together and move as one entity. Furthermore, two different bunched modes exist. Both modes have larger velocities than the corresponding single fluxon and the one with the highest velocity is the stable one. Erne and Parmentier ${ }^{3}$ found numerically that bunching may occur due to the effects of the boundaries even when the surface loss is absent. This has been confirmed by perturbation calculations. ${ }^{4}$ In the presence of damping resulting from the surface impedance in the superconducting films, it has been demonstrated numerically ${ }^{5}$ that fluxons moving close to the Swihart velocity posses a trailing "tail"' which is oscillating. The oscillating tail acts as a trap for neighboring fluxons leading to formation of multifluxon bound states. First experimental indication for soliton bunching has been found experimentally ${ }^{6}$ by measuring the emitted radiation in a linear junction. In the annular system, the bunching effect can break the symmetry and help the chain of solitons to overcome the dissipative losses. Their average velocity then becomes higher than that of a single soliton at the same bias current leading to a slightly larger voltage. Recently, it has been indicated by experiments, ${ }^{7}$ numerics, ${ }^{8}$ and theory ${ }^{9}$ that

\footnotetext{
${ }^{a)}$ Present address: Lehrstuhl Experimentalphysik II, Universität Tübingen, Auf der Morgenstelle 14, D-72076 Tübingen, Germany; Electronic mail: ivv@brahms.pit.physik.uni-tuebingen.de

${ }^{b}$ Permanent address: Institute of Solid State Physics, Russian Academy of Sciences, Chernogolovka, Moscow district, 142432, Russia.

${ }^{c}$ Present address: Institut für Schicht-Und Ionentechnik, Forschungszentrum Jülich (KFA), D-52425 Jülich, Germany.
}

bunched multifluxons exist in annular geometry at the velocities close to the maximum velocity (the Swihart velocity) of the electromagnetic waves in the junction.

Soliton motion in long one-dimensional Josephson junctions is described by the perturbed sine-Gordon equation ${ }^{10}$

$$
\varphi_{x x}-\varphi_{t t}=\sin \varphi+\alpha \varphi_{t}-\beta \varphi_{x x t}+\eta,
$$

where $\varphi(x, t)$ is the space and time dependent phase difference between the two superconducting films. The spatial coordinate $x$ is normalized to the Josephson penetration depth $\lambda_{j}=\left(\hbar / 2 \mu_{0} e d J_{c}\right)^{1 / 2}$, and the time $t$ is normalized to the inverse plasma frequency $\omega_{0}^{-1}=\left(\hbar C / 2 e J_{c}\right)^{1 / 2}$. $J_{c}$ is the maximum pair current density, $d$ is the magnetic thickness of the barrier $\left(d=\lambda_{1}+\lambda_{2}+t_{0}\right)$, and $C$ is the capacitance per unit area. The parameter $\alpha$ is the damping constant arising from quasiparticle losses and $\alpha=G\left(\hbar / 2 e J_{c}\right)^{1 / 2}$, where $G$ is the shunt conductance per unit area. The parameter $\beta$ denotes surface losses in the electrodes and $\beta=\omega_{0} L / R_{s}$, where $L$ is the inductance per unit length, $R_{s}$ is the electrode surface resistance per unit length. The normalized bias current is $\eta=I / I_{c}$. For the annular junction the boundary conditions for Eq. (1.1) are periodic, i.e.,

$$
\varphi(\ell, t)=\varphi(0, t)+2 \pi n, \quad \varphi_{x}(\ell, t)=\varphi_{x}(0, t),
$$

where $\ell$ is the junction length in units of $\lambda_{j}$ and $n$ is the number of fluxons trapped in the junction. The annular LJJ is a topologically closed system so the number $n$ of fluxons is conserved. From perturbation theory ${ }^{10,1}$ the steady-state velocity of the soliton solution of Eq. (1.1) is given by

$$
\eta=\frac{4}{\pi} u \gamma(u)\left(\alpha+\frac{\beta}{3} \gamma^{2}(u)\right)
$$

provided the junction length $\ell$ is sufficiently large. Here, $u$ is the fluxon velocity normalized to the Swihart velocity and $\gamma(u)$ is the Lorentz contraction factor given by $\gamma(u)=\left(1-u^{2}\right)^{-1 / 2}$. The width of the soliton is proportional to $1 / \gamma(u)$. 
In the following, we shall study experimentally as well as numerically the effect of the fluxon bunching in an annular LJJ. Contrary to the method proposed in Ref. 7 which requires unique low temperature scanning electron microscope (LTSEM) facilities, we demonstrate here a new and much more accessible procedure for trapping fluxons in an annular Josephson junctions. The steps in the I-V curve corresponding to $n$ fluxons have been measured and normalized to the voltage of the $n=1$ step. For some of the multisoliton steps these measurements give good evidence for the existence of a crossover from a nonbunched dynamic state to a bunched state as the bias current is increased. Such a crossover was observed for the first time in Ref. 7 but its dependence on damping was not studied. Here, we investigate this behavior at different temperatures. The two loss terms $\alpha$ and $\beta$ depend strongly on the temperature and this leads to a strong influence of the temperature on the I-V characteristic. We have performed the numerical simulations using values of the two loss terms estimated from a best fit of Eq. (1.3) to the corresponding experimental curve at that temperature. The numerical simulations confirm the interpretation of the experiments that bunching takes place at the top of some of the multifluxon steps.

The paper is organized as follows. The experimental setup and the method for trapping fluxons in the Josephson ring are described in Sec. II. Experimental results are presented in Sec. III, and in Sec. IV, we present the numerical simulations using parameter values which are in accord with the experimental data. In Sec. V, we discuss our experimental results and explain them on the basis of the numerical simulations and the bunching model. Finally, Sec. VI contains the conclusion.

\section{EXPERIMENTAL SETUP}

The measurements have been performed on niobiumlead annular Josephson junctions of a design very close to the classical geometry ${ }^{1}$ [Fig. 1(a)]. We have used junctions with an additional $150 \mathrm{~nm} \mathrm{SiO}$ insulating layer consisting of two concentric rings which covered the edges of the $\mathrm{Nb}$ electrode. ${ }^{11}$ The tunnel Josephson barrier was formed in the window between two $\mathrm{SiO}$ rings. The spatially resolved investigation using LTSEM showed a high homogeneity of the tunnel barrier of these window-type annular junctions. ${ }^{7}$ The $\mathrm{Nb}$ film of $200 \mathrm{~nm}$ thickness served as the base electrode. The tunnel barrier, grown by plasma oxidation of $\mathrm{Nb}$, was covered by a $350 \mathrm{~nm}$ thick top $\mathrm{Pb}$ electrode. The structure was formed using photolithography. Two sets of annular junctions were made. One set containing 6 junctions with the radius $R=150 \mu \mathrm{m}$ and with the width of the tunnel barrier ring $W=10 \mu \mathrm{m}$. The second set also includes 6 junctions but with $R=100 \mu \mathrm{m}$ and $W=20 \mu \mathrm{m}$. The sample holder was placed in a vacuum can. The sample temperature was controlled using a carbon thermometer and a compact bifilarily wound heater glued close to the sample. The measurements were performed in the temperature from $5.0 \mathrm{~K}$ to $5.8 \mathrm{~K}$ stepping by $0.2 \mathrm{~K}$.

The keystone of this work is the method used for trapping solitons in annular LJJs. Using photolithography, on an additional glass substrate we deposited a one-turn niobium
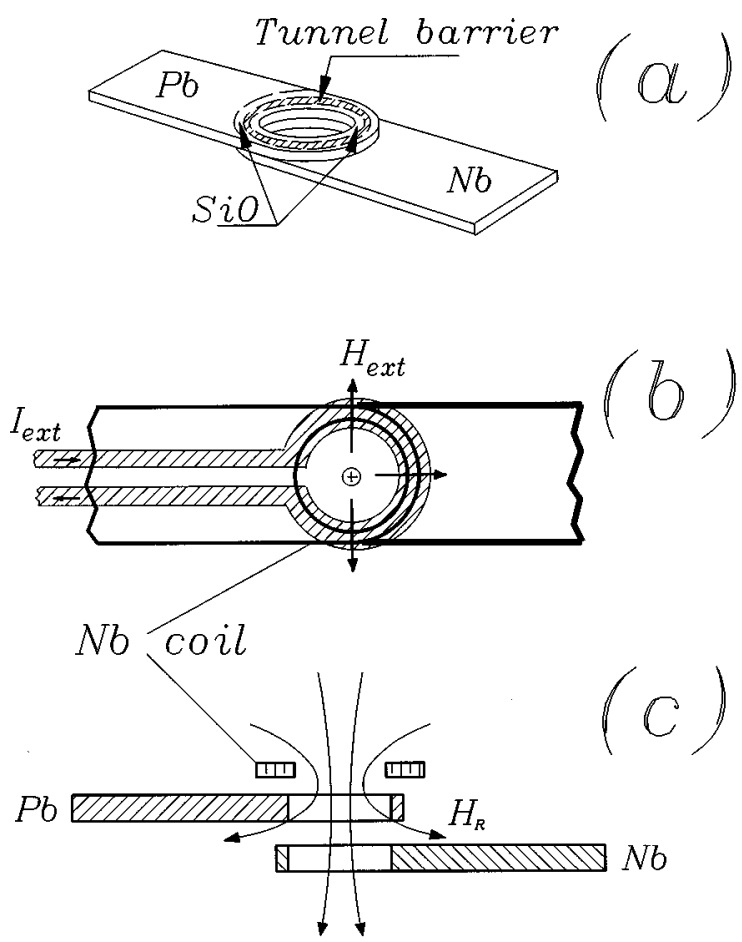

FIG. 1. (a) A sketch of the annular junction geometry used in the experiment. (b) Top view of the annular junction with the current coil placed above it. (c) Cross-section of the junction with the current coil.

coil with the same radius as the junction. ${ }^{12}$ The flipped glass substrate with the coil was placed above the silicon substrate with the annular junctions. The $\mathrm{Nb}$ coils of different junction were connected in series with each other and oriented coaxially with the junction rings [Fig. 1(b)]. The distance between two substrates during the experiment was several micrometers. Using a separate current source, we were able to apply the same current $I_{\text {ext }}$ through all the coils. In order to trap magnetic flux in the annular junctions the sample was heated up to a temperature $T_{1}$ slightly higher than the critical temperature $T_{c}^{\mathrm{Pb}}=7.2 \mathrm{~K}$ of lead and then cooled slowly in the presence of a certain constant current $I_{\text {ext }}$ in the $\mathrm{Nb}$ coils. The magnetic field $H_{\text {ext }}$ produced by the current $I_{\text {ext }}$ is expected to have a radial component $H_{R}$ in the plane of the tunnel barrier (Fig. 1(c)). During the cooling procedure and at $T \approx T_{c}^{\mathrm{Pb}}$, some magnetic flux produced by the field $H_{R}$ remained trapped in the tunnel barrier area of the junction. ${ }^{11}$ At $T=T_{0}<T_{c}^{\mathrm{Pb}}$, the current $I_{\mathrm{ext}}$ was switched off and the measurements of the $I-V$ characteristics were performed. The heater was used to remove trapped magnetic flux from the junction. Using this method, we were able to get different number of unipolar fluxons trapped in each junction. The number of fluxons $n$ depends on $I_{\text {ext }}$. In order to make the trapping procedure reproducible, heating and cooling processes were controlled by a computer.

\section{RESULTS}

We present here the results obtained for the junction with the radius $100 \mu \mathrm{m}$ and with the width of the tunnel barrier ring $W=20 \mu \mathrm{m}$. The measured critical current of the junction without trapped magnetic flux was $I_{c}=3.0 \mathrm{~mA}$. In 
calculating the critical current density, $70 \%$ of the current increase at the gap $I_{c}^{\prime}=0.7 \Delta I_{g}=3.45 \mathrm{~mA}$ was used instead of the measured critical current $I_{c}$ to eliminate effects of spatial variations of the bias current. ${ }^{13}$ The reduction factor of 0.7 accounts for the strong coupling effect in $\mathrm{Nb}-\mathrm{Pb}$ junctions. The Josephson penetration depth $\lambda_{j}$ was calculated to be approximately $100 \mu \mathrm{m}$ which corresponds to the length of the junction $L \approx 6.2 \lambda_{j}$ at $T=5.0 \mathrm{~K}$.

Theoretically, if fluxons are trapped in an ideally homogeneous annular junction the nondissipative critical current $I_{c}$ should be equal to zero. However, after the trapping procedure a certain critical current (typically $3 \%-5 \%$ of $I_{c}$ ) was observed. We suppose, that it is due to the a magnetic flux occasionally trapped in the superconducting electrodes. The most probable place for trapping flux is at the discontinuity region of the $\mathrm{Nb}$ coil where the current $I_{\mathrm{ext}}$ was injected into the coil (see Fig. 1(b)). It was possible to trap 1, 2, 3, and 4 fluxons without trapping of substantial magnetic flux (Abrikosov vortices) in the discontinuity region of the $\mathrm{Nb}$ rings. The measured $I-V$ characteristics with more than 4 fluxons had critical currents which are about $25 \%$ of the heights of the first steps. We anticipate that this behavior at large magnetic fields is due to the influence of trapped Abrikosov vortices which act as pinning centers for the fluxons.

The measured $I-V$ characteristics are symmetric around the origin $V=0$. Depending on the trapping current $I_{\text {ext }}$, different numbers $n$ of unipolar fluxons were found in the junction ring ( $n$ was approximately proportional to $I_{\mathrm{ext}}{ }^{11}$ ). As expected, the voltages of the resonant fluxon steps are quantized according to the formula $V_{n}=n \Phi_{0} \bar{c} / 2 \pi R$, where $\Phi_{0}$ is the magnetic flux quantum, $\bar{c}$ is the Swihart velocity, and $R$ is the inner junction radius.

Figure 2(a) shows the flux-flow parts of the experimental $I$ - $V$ curves at $T=5.0 \mathrm{~K}$ with 1 to 4 trapped fluxons. The step voltages are normalized to $n$, i.e., we display $V_{n} / n$. Since $V_{n} / n$ is proportional to the average fluxon velocity $u$, we can compare the dependence of $u$ on the external driving force $I \sim \eta$ for different fluxon densities. The voltages of the steps increases with increasing bias current and jumps to the gap voltage as the soliton velocity reaches $\bar{c}$. The steps were stable not only at lower currents but also for high currents just below the top of the steps. This compliments the previous experimental data ${ }^{7}$ where external noise influenced the stability at the top of the step. The global stability of the steps is confirmed by reproducibility of the results described below.

In Fig. 2(a), we observe small jumps (a sort of a crossover) to higher voltages on the steps 3 and 4 as the bias current is increased. On step 3 such a jump occurs at $I \approx 1.65 \mathrm{~mA}$ and on step 4 the jump is seen at $I \approx 0.95 \mathrm{~mA}$. The $I-V$ characteristics at the higher temperature $T=5.2 \mathrm{~K}$ are presented in Fig. 2(b). At this temperature, we observe a noticeable jump only in the fourth step at $I \approx 1.1 \mathrm{~mA}$.

Figure 3 shows the temperature dependence of the heights of the first four flux-flow steps. The height of the first step increases as the temperature increases whereas the heights of the higher order steps decreases. To our knowledge, the increase of the step height with temperature has never been observed experimentally before. Increasing the
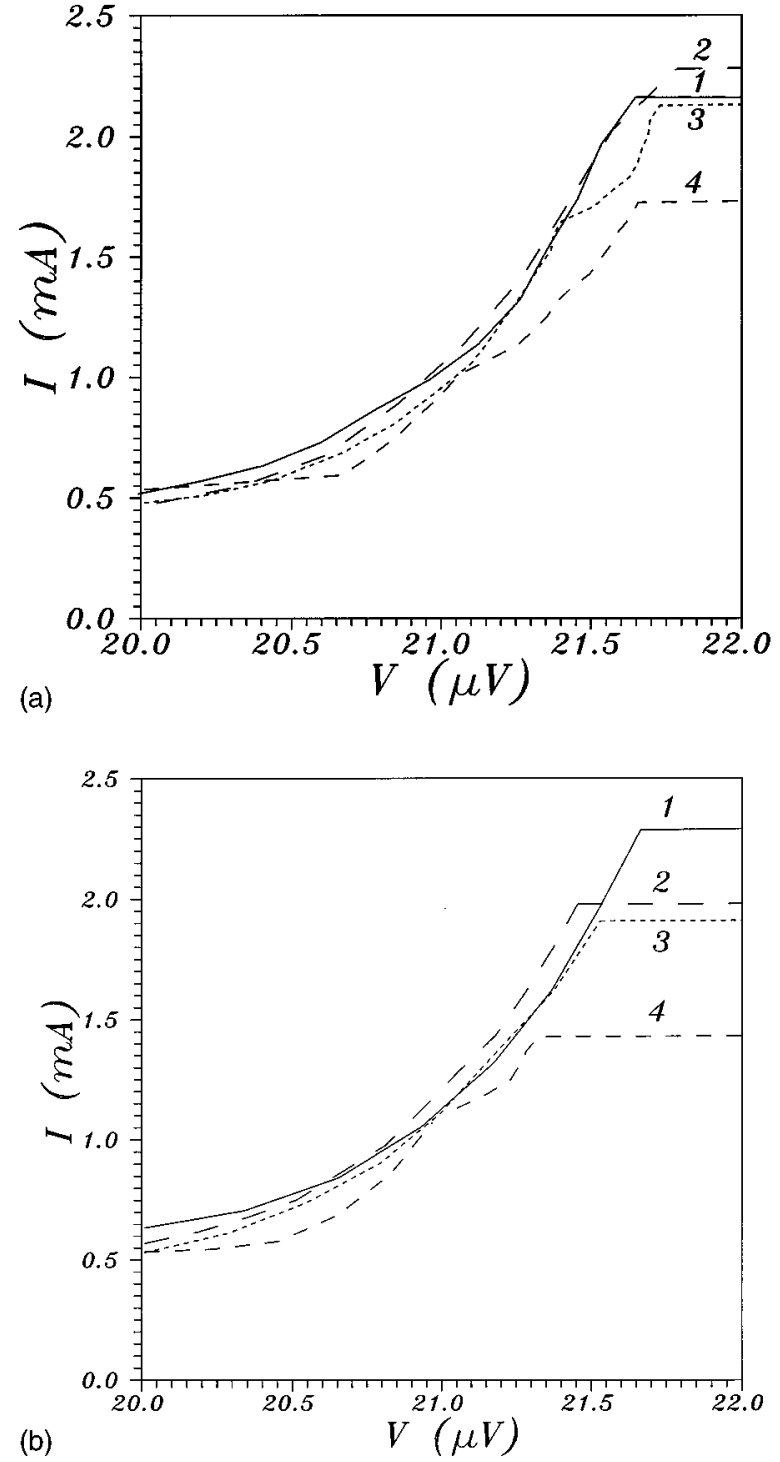

FIG. 2. I-V characteristics corresponding to different numbers of solitons in the annular Josephson junction (a) for $T=5.0 \mathrm{~K}$ and (b) for $T=5.2 \mathrm{~K}$. The voltage of the $n$-th step is divided by $n$. The numbers indicate the number of trapped solitons.

temperature leads to increasing $\alpha$ and $\beta$ losses. In conventional long linear Josephson junctions, the step height always decreases with increasing $\alpha$ and $\beta$ parameters. However, it should be mentioned that the question of step heights in current is not a simple one and is by no means settled. Some results in this direction were described numerically by Forest et al. ${ }^{14}$

For each measured value of the temperature in the range from $T=5.0 \mathrm{~K}$ to $5.8 \mathrm{~K}$, the parameters $\alpha$ and $\beta$ were determined. The experimentally obtained first fluxon step of the I-V curve was fitted to Eq. (1.3) using $\alpha, \beta$, and $V_{\text {step }}$ as fitting parameters. Parameters $\alpha_{\text {fit }}$ and $\beta_{\text {fit }}$ were obtained in a two-parameter fit after $V_{\text {step }}$ was separately determined by fitting to the Swihart velocity $\bar{c}$. As an example, in Fig. 4 we present the result of the fitting procedure for $T=5.0 \mathrm{~K}$ with $\alpha_{\mathrm{fit}}=0.02, \beta_{\mathrm{fit}}=0.0072$ and $V_{\text {step }}=21.9 \mu \mathrm{V}$. Figure 5(a) and 5(b) show a diagram of the fitted values $\alpha$ and $\beta$ versus 


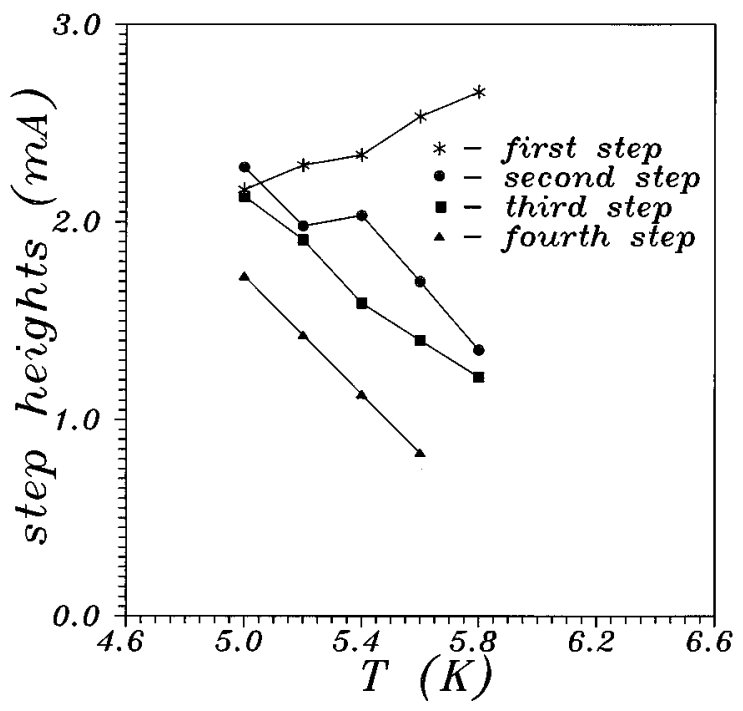

FIG. 3. Temperature dependence of the heights of the flux-flow steps for $n=1,2,3,4$. The numbers indicate the number of the step.

temperature. The step voltage is in the lower side of the $\left(\Delta_{\mathrm{Nb}}-\Delta_{\mathrm{Pb}}\right) / e$ voltage singularity in our junction made of two different superconductors $\mathrm{Nb}$ and $\mathrm{Pb}$. In this region, the quasiparticle resistance strongly depends on temperature, ${ }^{13}$ which is reflected by the strong temperature dependence of $\alpha_{\text {fit }}$. We note that the dependence of $\beta_{\text {fit }}$ on temperature is relatively weaker. The dependences on temperature of $\alpha_{\text {fit }}(T)$ and $\beta_{\text {fit }}(T)$ bear good resemblance to previous results. $^{13}$

\section{NUMERICAL SIMULATIONS}

The Eq. (1.1) with boundary conditions (1.2) were solved using a pseudospectral Fourier method. ${ }^{15}$ The parameters $\alpha$ and $\beta$ have been determined from the fitting of Eq. (1.3) to the experimental data as described in the previous section. In the temperature range considered here the normalized junction length $\ell$ was changed from $\ell=6.2$ for

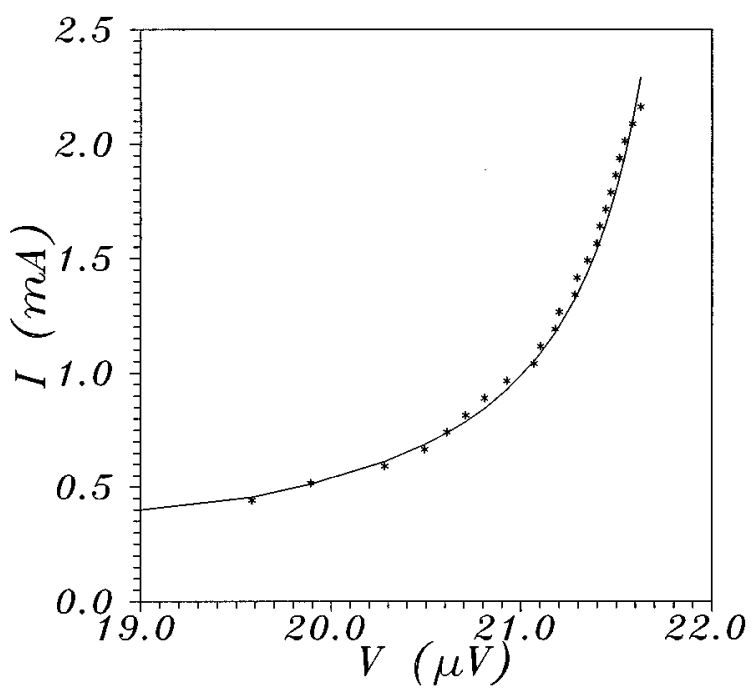

FIG. 4. Result of the fitting procedure for the first step at $T=5.0 \mathrm{~K}$ with $\alpha=0.02, \beta=0.0072$ and $V_{\text {step }}=21.9 \mu V$. *-experimental points.
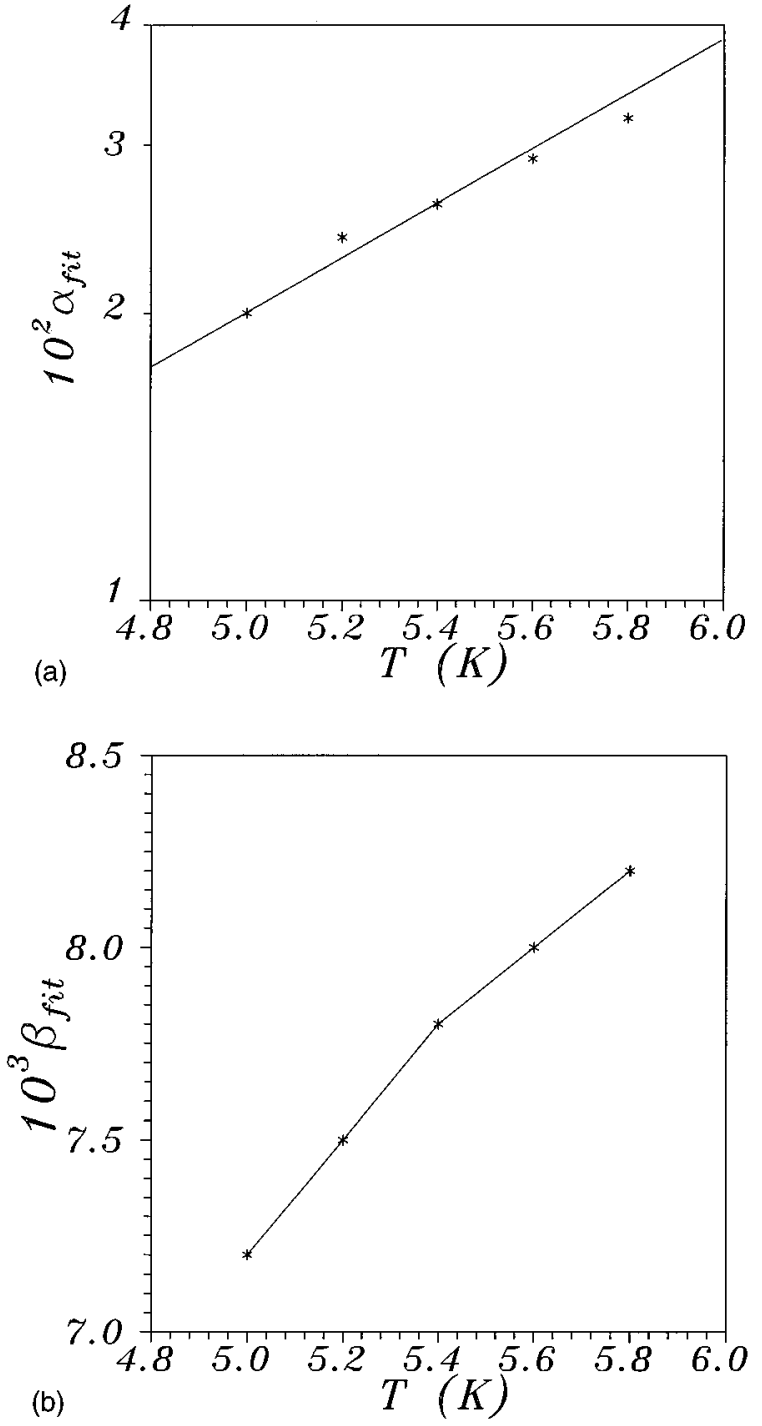

FIG. 5. (a) Temperature dependence of $\alpha_{\text {fit }}$. (b) Temperature dependence of $\beta_{\text {fit }}$.

$T=5.0 \mathrm{~K}$ till $\ell=5.7$ for $T=5.8 \mathrm{~K}$. The numerical simulations have been performed for $T=5.0 \mathrm{~K}$ and for $T=5.2 \mathrm{~K}$. At these two temperatures, the normalized lengths are $\ell=6.2$ and $\ell=6.1$, respectively. This change in the junction length has been neglected in the numerical simulations which has been performed using $\ell=6.2$. This neglect does not change the results qualitatively. In order to achieve a stationary dynamic state for each value of the bias current $\eta$, the integration was performed from 16 up to 1600 revolutions of the solitons. After that the average voltage $v$ $=\left\langle\phi_{t}\right\rangle$ was calculated using both temporal and spatial averaging. More specific we have calculated the voltage from $\left(J\left(t_{2}\right)-J\left(t_{1}\right)\right) /\left(t_{2}-t_{1}\right)$ where $J(t)=\left(\int_{0}^{\ell} \phi(x, t) d x\right) / \ell$. The reason for doing the spatial averaging is to improve accuracy in the voltage calculations in the case where the time difference $t_{2}-t_{1}$ is not an exact period of the oscillation.

The normalized $I-V$ characteristics $(\eta-V)$ have been calculated for different numbers of solitons $n$ in the system. Figures 6(a) and 6(b) show the results for the values of $\alpha$ and 

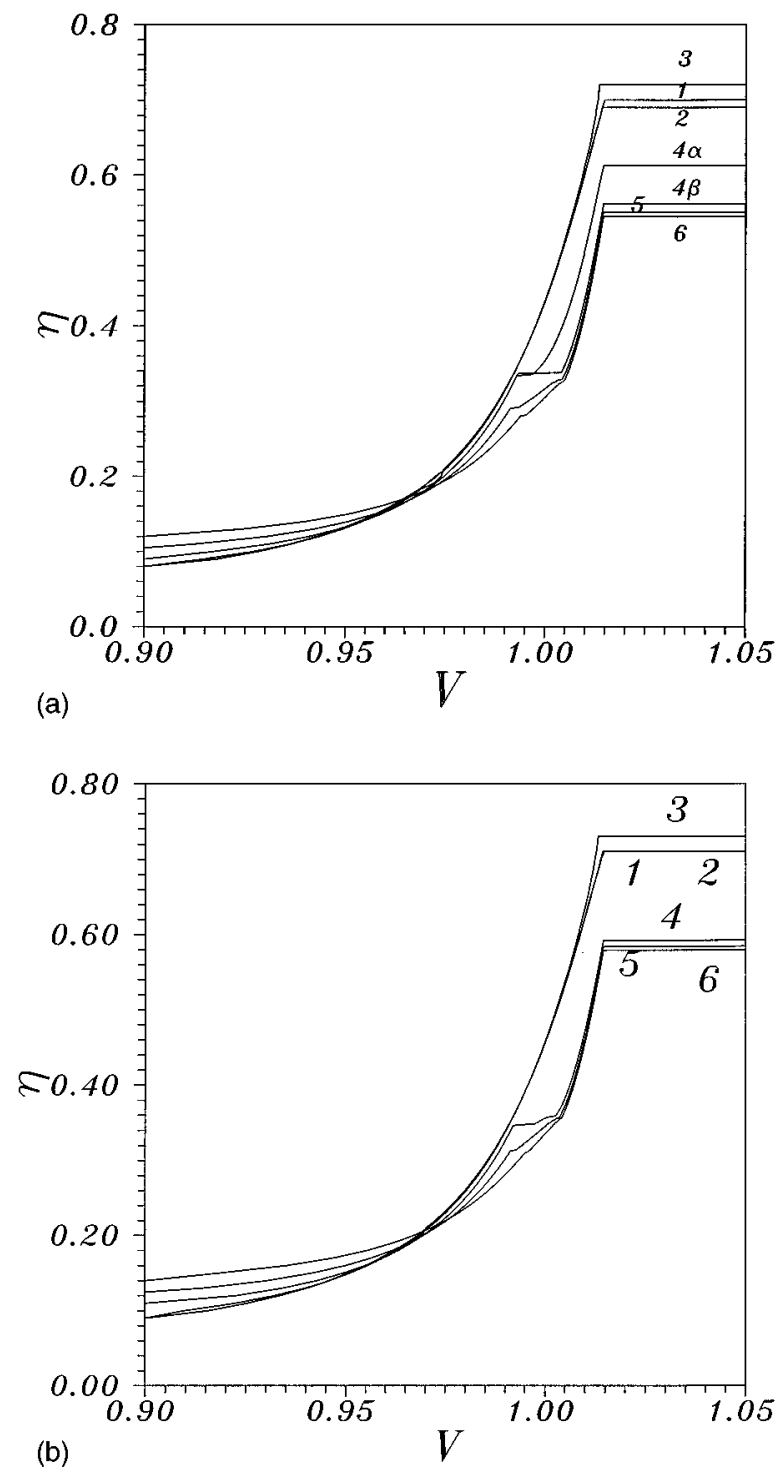

FIG. 6. The calculated I-V curves using the experimental determined parameter values (a) $\alpha=0.02$ and $\beta=0.0072$ corresponding to $T=5.0 \mathrm{~K}$; (b) $\alpha=0.024$ and $\beta=0.0075$ corresponding to $T=5.2 \mathrm{~K}$ with different numbers $n=1, \ldots, 6$ of trapped solitons. The voltage of the $n$-th step is divided by $n$. The numbers indicate the number of trapped solitons and on step $4, \alpha$ indicates the $2+2$ bunched state and $\beta$ indicates the 4 bunched state.

$\beta$ corresponding to $T=5.0 \mathrm{~K}$ and $T=5.2 \mathrm{~K}$, respectively. Apparently, this collection of curves looks quite similar to the experimental result. In a nice agreement with the experiment, the crossover to higher voltages is very pronounced for the step number $n=4$. However, no crossover is observed on step 3 but on the steps 5 and 6 . On step 4 at $T=5.0 \mathrm{~K}$ there are crossovers to two different states denoted by $4 \alpha$ and $4 \beta$, respectively.

For step 4 at $T=5.0 \mathrm{~K}$, the spatial magnetic field distribution $\varphi_{x}(x, t)$ is shown in Fig. 7 at three different points on the current-voltage characteristic. The three points are marked with crosses and labeled 1, 2, and 3. It is apparent that below the crossover point in the $\eta-V$ curve the four solitons are equidistantly spaced (the system has a translational symmetry in the $x$-direction with a period of $\ell / n)$ and

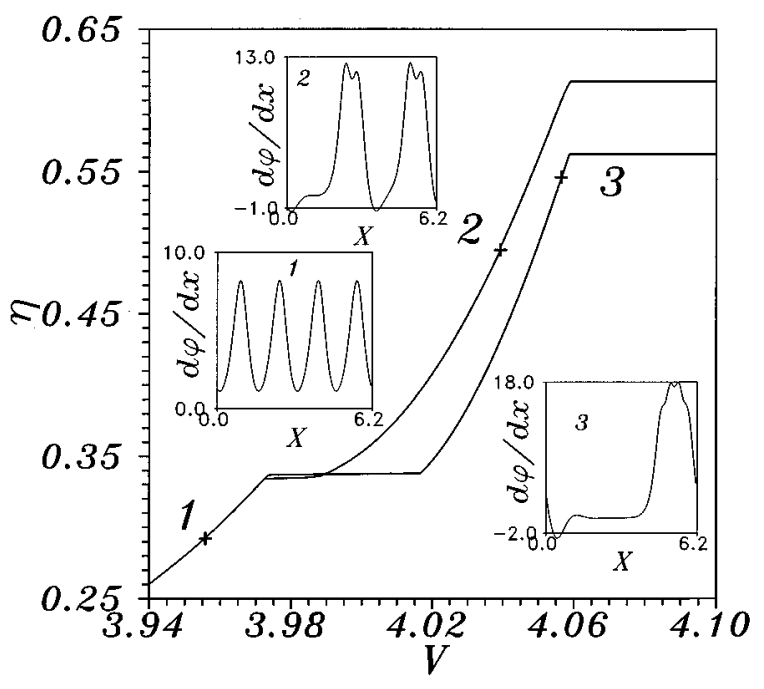

FIG. 7. Blow-up of the step number 4 (numerical simulations). The spatial distribution of the normalized magnetic field is shown at 3 points marked with crosses on the $I-V$ curve. (1) Nonbunched state below the crossover. (2) The $2+2$ bunched state. (3) The 4 bunched state.

accordingly the solitons are not bunched. However, above the small step we observe bunching of the solitons. At the mark labelled 2 the solitons are bunched together two and two. This configuration (branch $4 \alpha$ ) we shall denote the 2 $\times 2$ bunched state. In the second bunched state configuration (branch $4 \beta$ ), labelled by 3 , all four fluxons are merged together. This $1 \times 4$ configuration gives rise to a higher voltage than the $2 \times 2$ state, as expected. We did not identify such a splitting into two bunched states in the experiment. We estimate the possible voltage difference between the $2 \times 2$ and the $1 \times 4$ bunched configurations to be close to the voltaged resolution of our measurements. In addition, we cannot exclude the influence of the external noise on the stability of various bunched states. ${ }^{7}$

A similar crossover from nonbunched to bunched solitons are observed for the step 5 and 6 as the bias current $\eta$ is increased [see Figs. 6(a) and 6(b)]. Analogous behavior for the spatial magnetic field distribution $\varphi_{x}(x, t)$ is observed in the numerical simulation at $T=5.2 \mathrm{~K}$. These pictures strongly indicate that the crossover we observe in the experiments is due to bunching of solitons in the junction.

\section{DISCUSSION}

For a homogeneous annular LJJ the shape of the $I-V$ steps provides information about the soliton dynamics. The solitons travel through the junction with a velocity determined by a balance between the bias current and the losses in the junction in accordance with perturbation theory. ${ }^{10}$ The multisoliton behavior in homogeneous window-type annular LJJ has been studied recently in experiments ${ }^{7}$ and a comparison with existing multisoliton perturbation theory has been performed. ${ }^{16}$ In the present work, our aim was to investigate in more detail the dynamical behavior of solitons with velocity close to the Swihart velocity in a homogeneous annular LJJ. In this limit, the soliton dynamics cannot strictly be described within the framework of the perturbation theory. It 
is essential to emphasize that we restrict the experimental range to small damping $\alpha<0.1$ in our later consideration. Note that $\alpha=\bar{\alpha} \approx 0.6$ is the well-known critical viscosity associated with the Urabe bifurcation. ${ }^{17}$ Some finer details connected with larger $\alpha$ have been discussed by Burkov and Lifshitz. ${ }^{18}$

Let us discuss our experimental and numerical results assuming that the solitons are bunched at the top of the multisoliton steps. At the crossover from the equidistantly spaced solitons to the bunched soliton dynamic mode, a jump to higher voltages occurs in the numerical simulations. Accordingly, the corresponding voltage jump in the experiment we interpret as a result of a crossover from a nonbunched to a bunched dynamic state. The experiment [see Fig. 2(a)] shows a relative increase in the voltage which is $\sim 1.5 \%$ for the third step and $\sim 2.5 \%$ for the fourth step. The value of the voltage shift decreases with decreasing step number. This may be understood by analyzing the energies of the bunched and single soliton states. The energy gain of 4 bunched solitons is larger than that of 3 solitons compared to the single soliton. In the numerical simulations (Fig. 6) this relative voltage jump is about $1.25 \%$. Moreover, a transition to the bunched state was not observed experimentally for the step number 3 at $T=5.0 \mathrm{~K}$.

Returning to the experimental results in Figs. 2(a) and 2(b), we find that the transition point separating the bunched from the nonbunched state increases as the temperature increases. For $T=5.0 \mathrm{~K}$ we observe a crossover of the third step at $I \approx 1.65 \mathrm{~mA}$ and for the fourth step the crossover takes place at $I \approx 0.95 \mathrm{~mA}$. Increasing the temperature to $T=5.2 \mathrm{~K}$ the voltage shift due to bunching disappears on step number 3 , while the voltage shift on step number 4 appears at $I \approx 1.1 \mathrm{~mA}$. This behavior is mainly determined by the strong temperature dependence of the quasiparticle loss parameter in $\alpha$. Qualitatively, the lower part of the currentvoltage steps approach asymptotically the McCumber curve and increasing the $\alpha$ parameter by raising the temperature leads to a change of the slope of the McCumber curve. Hence, the background of the steps increase. This fact is clearly seen in the lower part of the steps calculated at different temperatures (Fig. 6). If only the influence of the $\beta$ parameter on bunching is taking into account, it have been shown that the criterion for bunching is ${ }^{9}$

$$
1-u_{\text {bunch }}{ }^{2}=\left(\frac{\beta^{2}}{2}\right)^{1 / 3}
$$

where $u_{\text {bunch }}$ is normalized to $\bar{c}$. For soliton velocities below $u_{\text {bunch }}$ bunching cannot happen and for velocities above $u_{\text {bunch }}$ bunching is possible. With increasing temperature, the parameter $\beta$ is increased and $u_{\text {bunch }}$ is decreased. However, in our case the parameter $\alpha$ is also changing and we cannot exclude an effect of the $\alpha$-term on bunching.

\section{CONCLUSION}

A new simple method has been realized by which one can easily insert individual fluxons and antifluxons into an annular Josephson junction in a controlled fashion. The quasiparticle and surface loss parameters have been determined as function of temperature by fitting experimental measured $I$ - $V$ characteristics to the formula in (1.3). Those temperature dependent loss parameters has been used in direct numerical simulations of the fluxon dynamics in the annular junction. For some steps, the experiments show a sharp voltage shift as the external bias current is increased. In accordance with the experiments a similar shift is seen in the numerical simulations. The calculated spatial magnetic field distributions indicate that the voltage shift results from a transition from a nonbunched to a bunched multifluxons dynamic state.

\section{ACKNOWLEDGMENTS}

We are grateful to B. A. Malomed and S. E. Burkov for stimulating discussions. We thank V. K. Kaplunenko for the layout design using his " $Z$ "'-topological compiler program. One of us (I.V.V.) gratefully acknowledges partial support from the Danish Research Academy. The present work was also supported by the NATO Linkage Grant No. LG 920672.

\footnotetext{
${ }^{1}$ A. Davidson, B. Dueholm, B. Kryger, and N. F. Pedersen, Phys. Rev. Lett. 55, 2059 (1985); A. Davidson, B. Dueholm, and N. F. Pedersen, J. Appl. Phys. 60, 1447 (1986).

${ }^{2}$ P. S. Lomdahl, O. H. Sørensen, and P. L. Christiansen, Phys. Rev. B 25, 5737 (1982); W. J. Johnson, Ph.D. thesis, University of Wisconsin, 1968 (unpublished).

${ }^{3}$ S. N. Erne and R. D. Parmentier, J. Appl. Phys. 52, 1091 (1981).

${ }^{4}$ V. I. Karpman and N. A. Ryabova, Phys. Lett. A 85, 251 (1981); V. I. Karpman, N. A. Ryabova, and V. V. Solovev, Sov. Phys. JETP 54, 705 (1981).

${ }^{5}$ A. Davidson, N. F. Pedersen, and S. Pagano, Appl. Phys. Lett. 48, 1306 (1986).

${ }^{6}$ B. Dueholm, O. A. Levring, J. Mygind, N. F. Pedersen, O. H. Sørensen, and M. Cirillo, Phys. Rev. Lett. 46, 1299 (1981).

${ }^{7}$ A. V. Ustinov, T. Doderer, R. P. Huebener, N. F. Pedersen, B. Mayer, and V. A. Oboznov, Phys. Rev. Lett. 69, 1815 (1992).

${ }^{8}$ M. P. Sørensen, B. A. Malomed, A. V. Ustinov, and N. F. Pedersen, Physica D 68, 38 (1993).

${ }^{9}$ B. A. Malomed, Phys. Rev. B 47, 1111 (1993).

${ }^{10}$ D. W. McLaughlin and A. C. Scott, Phys. Rev. A 18, 1652 (1978).

${ }^{11}$ I. V. Vernik, V. A. Oboznov, and A. V. Ustinov, Phys. Lett. A 168, 319 (1992).

${ }^{12}$ The idea of using the current coils on a separate substrate for this experiment was suggested by V. P. Koshelets.

${ }^{13}$ N. F. Pedersen and D. Welner, Phys. Rev. B 29, 2551 (1984).

${ }^{14}$ M. G. Forest, S. Pagano, R. D. Parmentier, P. L. Christiansen, M. P. Sørensen, and S. P. Sheu, Wave Motion 12, 213 (1990).

${ }^{15}$ F. If, M. P. Sørensen, and P. L. Christiansen, Phys. Lett. A 100, 68 (1984).

${ }^{16}$ P. M. Marcus and Y. Imry, Solid State Commun. 33, 345 (1980).

${ }^{17}$ M. Urabe, J. Sci. Hiroshima Univ. A 18, 379 (1955).

${ }^{18}$ S. E. Burkov and A. E. Lifshitz, Wave Motion 5, 197 (1983); Phys. Lett. A 106, 71 (1984).
} 